\title{
Formulation and Optimization of Atovaquone Micronized Suspension by Top-down Method
}

\author{
Harsha Kathpalia, Sukanya Das, Supriya Shidhaye* \\ Department of Pharmaceutics, Vivekanand Education Society's College of Pharmacy, Affiliated to University of Mumbai, Mumbai, \\ Maharashtra, INDIA.
}

\begin{abstract}
Aim: The present work aimed to tackle the solubility issue of a Biopharmaceutics Classification System (BCS) II drug, Atovaquone. Methods: Formulation of micronized suspension of atovaquone was optimized by employing a $3^{2}$ full factorial design keeping poloxamer 188 (wetting agent) and phospholipon $90 \mathrm{H}$ (stabilizer) as the influential variables affecting the dependent variables viz. particle size and polydispersity index (Pdl). Selected formulations from the optimized design space were further evaluated for dissolution and the formulation exhibiting maximum dissolution within 120 min was selected. The optimized batch consisting of atovaquone (15\%), poloxamer 188 (2\%), phospholipon $90 \mathrm{H}(1 \%)$, glycerin $(10 \%)$ in the aqueous vehicle was microfluidized. Results: The particle size of resultant suspension was $Z_{\text {avg }}=1313 \pm 40 \mathrm{~nm}$ and Pdl $=0.1 \pm 0.05$. The zeta potential of the final micronized suspension was $0.0555 \mathrm{mV}$ which indicated steric stabilization of the particles. The micronized suspension was adsorbed on a mixture of Kollidon CL SF and Aerosil 200 (2:1) and then dried and passed through 40\# sieve. Conclusion: The solid-state characterization of the formulation revealed that the crystalline nature of the drug was retained after the milling process. The saturation solubility and in-vitro dissolution of adsorbed micronized suspension were 2.3-fold times enhanced as compared to the pure drug suspension due to a reduction in particle size. The top-down approach can be employed to improve the poor aqueous solubility of atovaquone.
\end{abstract}

Key words: Atovaquone, Micronized suspension, Microfluidization, Full factorial design, Solubility enhancement.

\section{INTRODUCTION}

Enhancing the aqueous solubility is the most challenging phase of the drug development of a BCS class II drug. Atovaquone is a highly lipophilic $(\log p=5.8)$ antiprotozoal agent. Although it is acidic, the high $\mathrm{pKa}$ (9.1) renders it essentially neutral at physiological $\mathrm{pH}$. Atovaquone has extremely low aqueous solubility (less than $0.1 \mu \mathrm{g} / \mathrm{ml}$ ). The adult dose of Atovaquone is $1000 \mathrm{mg} /$ day for the treatment of malaria and 1500 $\mathrm{mg} /$ day for Pneumocystis pneumonia. In humans, the mean absolute bioavailability of atovaquone from Malarone tablets is merely $21 \%$ in the fed state and in the fasted state this is further reduced to $5-10 \%$. The bioavailability of atovaquone suspension is approximately $47 \%$ with food and without food, it is reduced to $23 \% .^{1-3}$

The absorption of atovaquone is solubility dependent. The salt formation, solubilization using various surfactants and particle size reduction have commonly been used to increase the dissolution rate and thereby bioavailability of such drugs. ${ }^{4,5} \mathrm{Few}$ approaches such as antisolvent precipitation based nanosuspension, ${ }^{5}$ solid lipid nanoparticles, ${ }^{6}$ Cyclodextrin complexation, ${ }^{7}$ liposomes, ${ }^{8}$ and nanocapsules ${ }^{9}$ have been successfully implemented to improve the solubility of atovaquone. However, all these researches suffer from a drawback of low drug loading. To overcome this drawback, the micronized suspension provides a means
Submission Date: 01-11-2019; Revision Date: 12-04-2020; Accepted Date: 11-07-2020

DOI: 10.5530/ijper.55.1.11 Correspondence: Dr. Supriya Shidhaye Principal, Vivekanand Education Society's College of Pharmacy, Hashu Advani Memorial Complex, Chembur, Mumbai-400 074, Maharashtra, INDIA.

Phone: +91 022-255439225 E-mail: supriya.shidhaye@ ves.ac.in

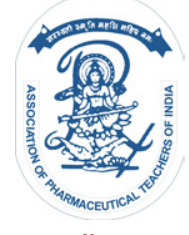

www.ijper.org 
to increase the drug loading without the interference of toxic side effects caused by co-solvents.

Micronized suspensions can be prepared by two methods namely, bottom-up technology and topdown technology. Bottom-up technologies include precipitation, microemulsion approach and melt emulsification. Top-down technology involves the fragmentation of larger particles into smaller, examples of which are high-pressure homogenization and milling methods. ${ }^{10}$ One such top-down method of size reduction is microfluidization. Particles dispersed in the suspension pass through the interaction chambers at high pressure as jet streams. The particle size reduction takes place when the particles flowing through this jet stream collide with each other. The top-down approach, unlike the bottom-up approach, does not require a solvent for processing of the formulation. ${ }^{11}$

The major advantage of micronized suspension is the enhanced dissolution achieved due to reduced particle size and increased specific surface area of the drug particles. A reduction in particle size affects the stability of the drug by particle agglomeration upon storage. Hence their transformation into solid products is often required to maintain its physical stability. This can be achieved by techniques such as freeze-drying, spraydrying, pelletization, or granulation. Recently adsorption onto silicates in amorphous forms or as solutions has gained substantial importance in pharmaceutical research. Silicates can absorb liquids as much as thrice their weight. Some of the examples of silicates are Aerosil $^{\circledR} 200$, Neusilin ${ }^{\circledR}$ UFL2, Neusilin ${ }^{\circledR}$ US2, Sipernat ${ }^{\circledR}$ 22, Sylysia ${ }^{\circledR} 350$, Zeopharm ${ }^{\circledR} 600 . .^{12,13}$

In the present study, atovaquone micronized suspension was prepared by a three-stage top-down processing

- Wet milling using trituration method

- Probe sonication

- Microfluidization

This three-stage processing was used to reduce the particle size of atovaquone from 9 microns to a particle size range of $1200-1400 \mathrm{~nm}$. To maintain this particle size and prevent particle size growth, the micronized suspension was further adsorbed on a substrate to convert it into a solid dosage form. Poloxamer 188 was used as a wetting agent and Phospholipon $90 \mathrm{H}$ as a stabilizer. Phospholipon is a phospholipid that has been studied to have an impact on solubility of waterinsoluble drugs. ${ }^{14}$

\section{MATERIALS AND METHODS Materials}

Atovaquone was received as a gift sample from Glenmark Pharmaceuticals Ltd., Mumbai, India.
Poloxamer (Kolliphor ${ }^{\circledR} \mathrm{P} 188$ ), crospovidone (Kollidon ${ }^{\circledR}$ CL SF) and polyethoxylated castor oil (Kolliphor ${ }^{\circledR}$ EL) were received from BASF, Navi Mumbai, India. Hydrogenated phospholipid (Phospholipon ${ }^{\circledR} 90 \mathrm{H}$ ) was obtained as a gift sample from Lipoid GmbH, Germany. Colloidal silicon dioxide $\left(\right.$ Aerosil ${ }^{\circledR} 200$ ) was obtained from Evonik, India. All other chemicals and reagents were purchased from LobaChemie, India.

\section{Pre-formulation studies and screening of excipients}

Equilibrium solubility studies of atovaquone were carried out employing water, various buffer solutions and solutions of surfactants like tween 80, sodium lauryl sulphate, Kolliphor EL buffered at different $\mathrm{pH}$ values. The equilibrium solubility studies were also carried out in a $10 \%$ surfactant solution in water with surfactants like poloxamer, Solutol HS 15, Phospholipon 90H, d- $\alpha$-Tocopheryl polyethylene glycol 1000 succinate (Kolliphor $^{\circledR}$ TPGS), polyvinyl pyrrolidone $\left(\right.$ Kolliphor $^{\circledR}$ $\mathrm{K} 30)$ and tween 80. The excess amount of the drug was added to $10 \mathrm{ml}$ of each solution in screw-capped vials and vortex mixed for $30 \mathrm{~s}$ (Vortex mixer, Remi, Mumbai, India). The sealed vials were shaken in an orbital shaker incubator (Hally Instruments, India) at $37^{\circ} \mathrm{C}$ at 200 rpm for $48 \mathrm{hr}$. The samples were centrifuged (REMI 120C, REMI Labs, India) at 15,000 rpm for $5 \mathrm{~min}$ and then filtered through a $0.22 \mu \mathrm{m}$ nylon syringe filter. The filtrates were assayed in triplicates by validated HPLC method.

\section{Selection of thickener/stabilizer}

Kolliphor K 30, d- $\alpha$-tocopheryl polyethylene glycol 1000 succinate (TPGS), Phospholipon $90 \mathrm{H}$ were evaluated as stabilizers/thickeners at $1 \%$ concentration to keep the drug particles suspended in aqueous medium after wetting with poloxamer P188. Micronized suspensions at $15 \%$ atovaquone concentration with these stabilizers were prepared in presence of poloxamer P188 and evaluated for sedimentation behavior and ease of redispersibility.

\section{Preparation of atovaquone suspension}

The drug concentration in all formulations was kept constant at $15 \% \mathrm{w} / \mathrm{w}$. The drug was dispersed in an aqueous solution of $2-10 \%$ poloxamer 188 and $1-5 \%$ Phospholipon $90 \mathrm{H}$ with trituration. Glycerine (10\%) was added as viscosity building agent and volume was made up with purified water. This suspension was homogenized at 10,000 $\mathrm{rpm}$ for 10min (Ultraturrax T25 with S25N-10G dispersing element, IKA-Werke $\mathrm{GmbH}$ and Co.KG, Staufen, Germany) and then probe sonicated for $10 \mathrm{~min}$ (Oscar Ultrasonic Processor 
Sonapros PR-250, Mumbai, India) using $3 \mathrm{~mm}$ horn at $30 \%$ variac for further particle size reduction.

\section{Optimization of atovaquone suspension}

Risk assessment was carried out for screening the critical formulation variables and it was observed that levels of both surfactants (poloxamer 188), as well as a stabilizer (phospholipon $90 \mathrm{H}$ ), were critical. A $3^{2}$ full factorial design was used to statistically optimize the formulation factors in the development of micronized suspension and for quantitatively expressing the effect of influential formulation variables viz. surfactant and stabilizer concentration on the response variables viz: the critical quality attributes like particle size expressed as Z-average diameter of the particles in $\mathrm{nm}$ and polydispersity index (PdI). Table 1 presents the description of a total of 9 experimental runs at various levels of formulation variables and the design matrix as per full factorial design. All the prepared formulations were characterized for the envisioned critical quality attributes, viz. particle size $\left(Z_{\text {avg }}, n m\right)$ and polydispersity index and optimized formulations were selected.

Statistical analysis was performed using Design Expert (Version 8.1, Stat-EASE INC., Minneapolis, MN, USA) software. For this study, the multiple linear regression analysis was applied to fit the model with a full second-order quadratic polynomial equation with added interaction terms to correlate the response variables with the evaluated variables using DesignExpert software. One-way ANOVA was carried out by analyzing the model parameters like model $p$-value, coefficient of correlation and predicted error sum of squares. The response surface analysis was carried out using the 3D-response surface plots and contour plots. The optimized formulation was selected from the design space overlay plot based on the minimum particle size and polydispersity index. Numerical optimization was carried out using the desirability function by studying the critical material attributes to meet the desired goals for each critical quality attribute.

\section{Microfluidization of Atovaquone suspension}

The particle size of the probe-sonicated suspension was further reduced by microfluidization (Microfluidizer

\begin{tabular}{|c|c|c|c|c|c|c|c|c|c|}
\hline Ingredient & 1 & 2 & 3 & 4 & 5 & 6 & 7 & 8 & 9 \\
\hline $\begin{array}{c}\text { Poloxamer } \\
188(\%)\end{array}$ & 2 & 2 & 2 & 6 & 6 & 6 & 10 & 10 & 10 \\
\hline $\begin{array}{c}\text { Phospholipon } \\
90 \mathrm{H}(\%)\end{array}$ & 1 & 3 & 5 & 1 & 3 & 5 & 1 & 3 & 5 \\
\hline
\end{tabular}

LM20, Microfluidics Corp, Massachusetts, equipped with Z-chamber) of the suspension at a temperature $15-20^{\circ} \mathrm{C}$. The particle size reduction during various cycles of microfluidization was monitored using a visible microscope at $40 \mathrm{X}$ magnification, the endpoint is no distinctly measurable particles. The suspension was passed 20 times through the microfluidizer under 1500 bars pressure and 10 times under 2000 bars pressure. Based on the requirement of particle size, the number of cycles of microfluidization and pressure was optimized.

\section{Adsorption studies}

Adsorption studies were carried out using Kollidon CL SF and Aerosil 200 as the adsorbent, in the ratio 2:1. The required amount of adsorbent was spread in a porcelain dish and the microfluidized micronized suspension was poured over the adsorbent uniformly to saturate it till wet (mixing was done as required to ensure uniform adsorption). After partial drying remaining micronized suspension was added and allowed to air dry. The dried adsorbed suspension was passed through $40 \#$ sieve.

\section{Characterization of micronized suspension}

The micronized suspension was diluted 10 times with Millipore water, filled in the cuvette and analyzed. The adsorbed micronized suspension was diluted in water to achieve a concentration of $150 \mathrm{mg} / \mathrm{ml}$. This reconstituted suspension was checked for ease of redispersibility. The particle size, PdI and zeta potential were determined by diluting the reconstituted suspension 10 times with Millipore water and analyzing it using Malvern Zeta sizer (Nano ZS90, Malvern Instruments, Malvern, UK).

The adsorbed powder was dissolved in methanolic sodium hydroxide and further diluted with methanol:water (50:50). The solution was analyzed for drug content using HPLC at $220 \mathrm{~nm}$. The saturated solution of adsorbed micronized suspension in water was shaken for $48 \mathrm{hr}$. to determine the solubility. The solution was centrifuged, filtered and analyzed using HPLC at $220 \mathrm{~nm}$.

The powder X-ray Diffraction (XRD) pattern was recorded using an X-ray diffractometer (X'PertPRO MPD, PANalytical, Netherland) at $40 \mathrm{kV}, 30 \mathrm{~mA}$ at a scanning rate of $0.02^{\circ} / \mathrm{min}$ at the diffraction angle $2 \theta$ over the range 10-80 using copper (as an anode) radiation of wavelength $1.5405 \mathrm{~A}^{\circ}$. The thermal characteristics of adsorbed nanosuspension and pure drug samples were evaluated using differential scanning calorimeter (DSC 6220, SII Nanotechnology, SEIKO). Alumina was used as a reference and the thermogram 
of the drug was scanned from $30^{\circ} \mathrm{C}$ to $300^{\circ} \mathrm{C}$ with a rate of increase in temperature of $10^{\circ} \mathrm{C}$ per min.

\section{In vitro dissolution studies}

Dissolution studies were carried out for a sample of adsorbed micronized suspension and pure drug suspension in USP XXIV, Apparatus 2 paddle method (M/s LabIndia, DS A/S 8000, Mumbai, India) employing $1000 \mathrm{~mL}$ of $2 \%$ Kolliphor EL in water as the discriminatory medium providing sink conditions at $50 \mathrm{rpm}$ and $37 \pm 0.5^{\circ} \mathrm{C} \cdot{ }^{15}$ An aliquot of the sample $(5 \mathrm{~mL})$ was periodically withdrawn at suitable time intervals, followed by replacement with an equivalent amount of fresh dissolution medium. The aliquot was filtered through 0.22 micron and diluted with methanolic sodium hydroxide and further, the volume was made with methanol:water (50:50) and analyzed using the HPLC method.

\section{RESULTS AND DISCUSSION}

\section{Equilibrium solubility studies}

Among various mediums explored for equilibrium solubility studies, the maximum solubility for atovaquone was observed in 40\% IPA in phosphate buffer $\mathrm{pH} 8$ (Figure 1), which is the official medium as per OGD guidelines of the US FDA for atovaquone tablets. But it is not a discriminatory medium for dissolution; hence $2 \%$ Kolliphor EL solution in water showing significant solubility of atovaquone $(50 \mu \mathrm{g} / \mathrm{mL})$ was selected as a discriminatory organic solvent-free medium for dissolution studies. Similarly, amongst various surfactants employed, the minimum solubility and maximum wettability was obtained in poloxamer 188 (Figure 2) and it was selected as the wetting agent (Table 2). Poloxamer 188 has been used

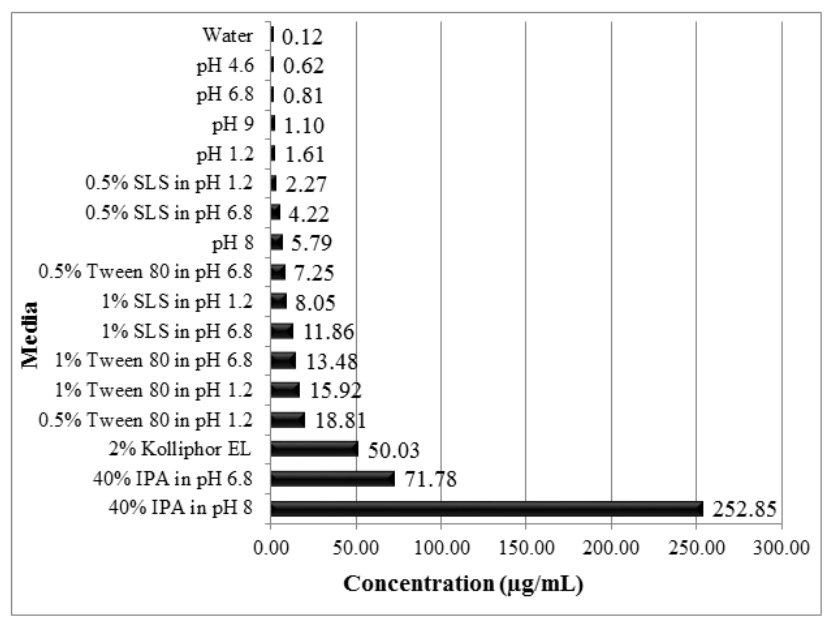

Figure 1: Equilibrium solubility study in various media. in marketed suspension (Mepron) as a wetting agent in $0.5 \%$ concentration and is also listed as one of the inactive ingredients in Malarone tablets. Poloxamer 188 is an amphiphilic nonionic block polymer and its hydrophobic polypropylene oxide segment adsorbs on the surface of the drug and its hydrophilic polyethylene oxide segment provides steric hindrance to prevent particle aggregation. The hydrophobicity of atovaquone molecules affects its dispersibility, poloxamer act as a wetting agent and assist in dispersion. Thereafter, it acts as a non-ionic stabilizer.

\section{Selection of thickener/stabilizer}

Several batches were prepared using the standard preparation method (containing only the atovaquone and poloxamer) in the absence of stabilizer. From microscopic views (not shown), agglomeration of nanoparticles was observed without the addition of stabilizer. Based on sedimentation behavior and ease of redispersibility, Phospholipon $90 \mathrm{H}$ was selected as the stabilizer. The suspension showed a thick floc

\begin{tabular}{|c|c|}
\hline Table 2: Selection of a wetting agent. \\
\hline Surfactants & Wettability \\
\hline Sodium lauryl sulphate & +++ \\
\hline Tween 80 & ++ \\
\hline Poloxamer 188 & +++ \\
\hline Solutol HS 15 & + \\
\hline Poloxamer 407 & - \\
\hline Phospholipon $90 \mathrm{H}$ & - \\
\hline Kolliphor RH 40 & - \\
\hline Kolliphor EL 50 & - \\
\hline Kolliphor TPGS & - \\
\hline
\end{tabular}

+++ Excellent ++ Good + Not acceptable - Poor

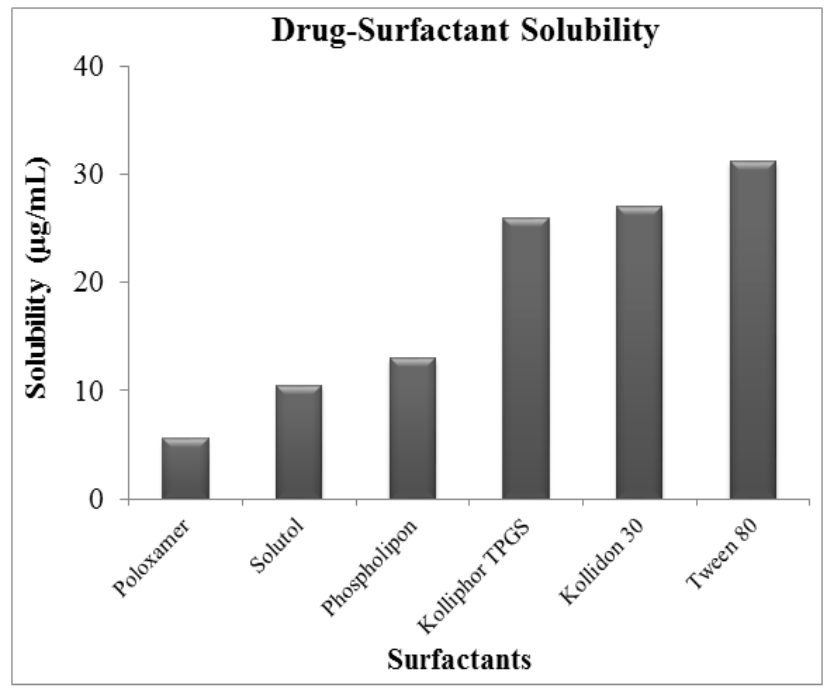

Figure 2: Equilibrium solubility study in surfactant solutions. 
without any sign of sedimentation and was easily redispersed in a single stroke. Thus, Phospholipon $90 \mathrm{H}$ was found to be an indispensable dispersing agent required in the preparation of well-dispersed homogenous suspension. Phospholipon $90 \mathrm{H}$ provides the phospholipid network to entrap the drug particles without forming strong bonds. The phospholipid offers steric hindrance that prevents particle size growth and even after sedimentation, the flocs easily redisperse on shaking. The concentration of Phospholipon is critical as higher concentration may affect the release of drug entrapped in the phospholipids. Non-ionic surfactants like Phospholipon $90 \mathrm{H}$ and Poloxamer tend to increase the solution viscosity, which in turn provides a barrier against aggregation.

\section{Preparation and optimization of atovaquone suspension}

With the consideration of the concentration Phospholipon $90 \mathrm{H}$ and Poloxamer 188 as the critical formulation parameter that will have an impact on the properties of the micronized suspension, a polynomial was fitted based on the difference in the values of predicted and adjusted $r^{2}$. On comparing with other models, it was suggested that the quadratic model for the particle size and 2F model for PdI is best suited due to the largest values of $r^{2}$ for all the responses.

The results of the experimental design for particle showed that the upward trend of wire mesh in the 3D surface plot was at a higher concentration of poloxamer 188 whereas phospholipon $90 \mathrm{H}$ did not have any effect on the particle size. On the contrary, the interaction of poloxamer 188 and phospholipon $90 \mathrm{H}$ had a significant effect on particle size. The factors $\mathrm{B}, \mathrm{AB}, \mathrm{B}^{2}$ are significant terms in this model, B (phospholipon $90 \mathrm{H}$ ), $\mathrm{AB}$ (Interaction of $\mathrm{A}$ and $\mathrm{B}$ ) and $\mathrm{B}^{2}$ (phospholipon $\left.90 \mathrm{H}^{2}\right)$ are significant model terms considering the $p<$ 0.05. The final mathematical equation of the fitted model for particle size is as follows

Particle size $=20.53+1.55 \mathrm{~A}-8.25 \mathrm{~B}+7.18 \mathrm{AB}-0.62 \mathrm{~A}^{2}$ $+10.74 \mathrm{~B}^{2}$

where A is Poloxamer 188 and B is Phospholipon $90 \mathrm{H}$ The results of the experimental design for PdI showed that the upward trend of the mesh of the $3 \mathrm{D}$ surface plot was at a high level of phospholipon $90 \mathrm{H}$ and low level of poloxamer 188. A relatively lower trend of mesh is seen at a higher level of Poloxamer 188 and a lower level of phospholipon $90 \mathrm{H}$. The interaction of poloxamer 188 and phospholipon $90 \mathrm{H}$ also has a significant effect on PdI. In this model, B (phospholipon 90H), AB (Interaction of $\mathrm{A}$ and $\mathrm{B}$ ) and $\mathrm{B}^{2}$ (phospholipon $90 \mathrm{H}^{2}$ ) are significant model terms. The final mathematical equation of the fitted model for PdI is as follows

$\mathrm{PdI}=26.13-2.0 \mathrm{~A}+2.47 \mathrm{~B}+12.3 \mathrm{AB}$

Where A is Poloxamer 188 and B is Phospholipon $90 \mathrm{H}$ Three-dimensional response surface plots and twodimensional contour plots are shown in Figure 3. To optimize all the responses with different targets, a graphical optimization technique by the overlay plot was used. The optimized formulation was obtained by applying constraints on dependent variable responses and independent variables. An overlay contour plot was plotted between two excipients, X1 and X2 which showed the region between the two set criteria, i.e. particle size which should be between $1000 \mathrm{~nm}$ to $2500 \mathrm{~nm}$ and polydispersity index which should be between 0.1 and 0.3. The yellow region in Figure 4 indicates the region of successful operating ranges.

Two batches were selected from the optimized region of the overlay plot (i) $2 \%$ poloxamer, $5 \%$ phospholipon $90 \mathrm{H}$ and (ii) $2 \%$ poloxamer, $1 \%$ phospholipon $90 \mathrm{H}$. These batches were prepared and studied for dissolution behavior. It was observed that a maximum $15.5 \%$ drug was dissolved in $2 \mathrm{~h}$ with $5 \%$ phospholipon $90 \mathrm{H}$ whereas $18.8 \%$ of the drug was dissolved in $2 \mathrm{~h}$ with $1 \%$ phospholipon $90 \mathrm{H}$. Hence formulation with $2 \%$ poloxamer and $1 \%$ phospholipon $90 \mathrm{H}$ was the optimized batch. Excess of phospholipon interfered with the drug release by forming a dense network in the aqueous medium. The formula for final optimized suspension is shown in Table 3.

\section{Microfluidization}

Microfluidization has tremendous potential to produce commercially viable products due to its ease of scalability. ${ }^{16}$ A patent of Glaxo Wellcome Inc., mentions the use of microfluidzation technique to bring the down the particle size of atovaquone in the range of 0.1 to $2 \mu \mathrm{m} .{ }^{17} \mathrm{It}$ is important for the formulation to pass through high shear homogenization using Ultra-Turrax and probe sonication to avoid jamming

\begin{tabular}{|c|c|c|c|}
\hline $\begin{array}{l}\text { Sr. } \\
\text { No }\end{array}$ & Components & Quantity & Role of excipients \\
\hline 1 & Atovaquone & $15 \%$ & $\mathrm{API}$ \\
\hline 2 & Poloxamer 188 & $2 \%$ & Wetting agent \\
\hline 3 & Phospholipon $90 \mathrm{H}$ & $1 \%$ & Stabilizer \\
\hline 4 & Glycerine & $10 \%$ & $\begin{array}{c}\text { Wetting and } \\
\text { suspending agent }\end{array}$ \\
\hline 5 & Purified water & q.s $100 \%$ & Vehicle \\
\hline
\end{tabular}


in the valve openings of microfluidizers. ${ }^{18}$ The final optimized batch was microfluidized after exposing it to high shear homogenization with Ultra-Turrax at $10,000 \mathrm{rpm}$ for $10 \mathrm{~min}$ and probe sonication for $10 \mathrm{~min}$. Microfluidized samples were observed under a microscope at various steps of the microfluidization process as shown in Figure 5. Reduction in particle size was observed upto a limiting number of cycles and pressure and then further increase caused aggregation.

\section{Adsorption study}

The microfluidized micronized suspension was found to show aggregation after two days even at refrigerated conditions, hence the suspension was adsorbed onto the Aerosil-Kollidon CL SF mixture to prevent an increase in particle size. Air drying was the preferred method to avoid aggregation of particles. For example, if the nanoparticles are coated with polymeric surfactants such as poloxamers, drying at elevated temperature may lead to crystallization of the polymer, thereby compromising their ability to prevent aggregation. Drying can also create additional thermal stresses that may destabilize the particles. ${ }^{19}$

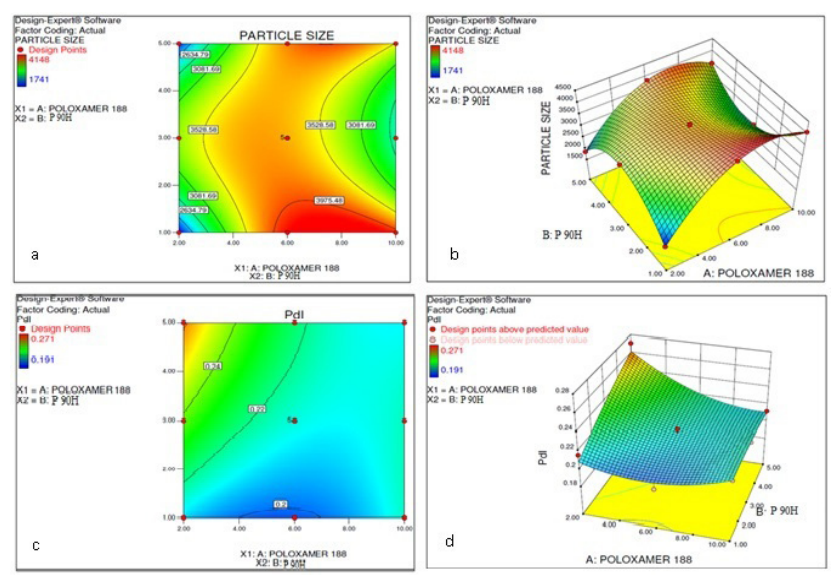

Figure 3: Contour plots for Particle size and Pdl.

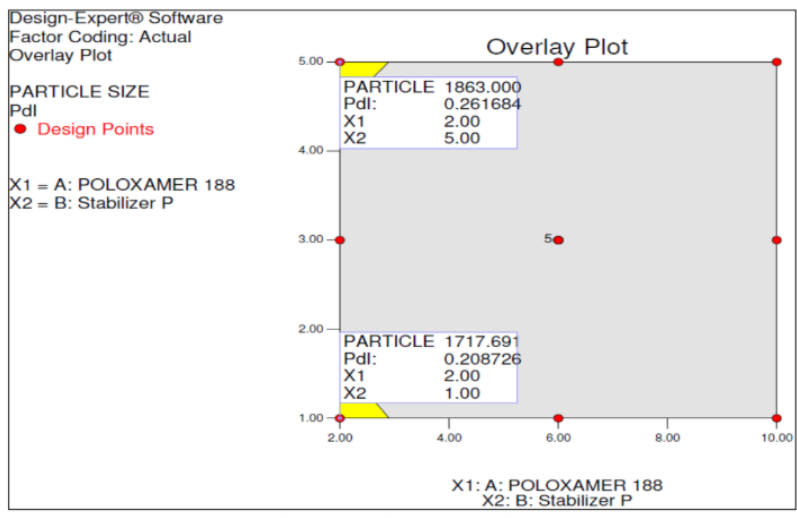

Figure 4: Overlay plot for Particle size and Pdl.

\section{Characterization of micronized suspension}

The particle size of the pure drug was 8-9 microns. Table 4 depicts the particle size of the suspension at various stages of preparation showing a progressive decrease in particle size with an increase in shear. The zeta potential value was $0.0555 \mathrm{mV}$ which indicates that the micronized suspension has steric stability and aggregation did not occur.

The adsorbed micronized suspension was easily and uniformly re-dispersed in water. A slight shift in the particle distribution to the higher particle size was observed. The mean particle size of the reconstituted powder particles was typically $30 \%$ higher than the mean

Table 4: Particle size and polydispersity index.

\begin{tabular}{|c|c|c|}
\hline $\begin{array}{c}\text { Stage of preparationof } \\
\text { micronized suspension }\end{array}$ & $\begin{array}{c}\text { Particle } \\
\text { size } \\
\mathbf{Z}_{\text {avg }}, \mathbf{n m}\end{array}$ & $\begin{array}{c}\text { Polydispersity } \\
\text { index }\end{array}$ \\
\hline Probe sonication & $1900 \pm 100$ & $0.500 \pm 0.05$ \\
\hline Microfluidization & $1313 \pm 40$ & $0.100 \pm 0.05$ \\
\hline Adsorption & $1700 \pm 200$ & $0.400 \pm 0.10$ \\
\hline
\end{tabular}

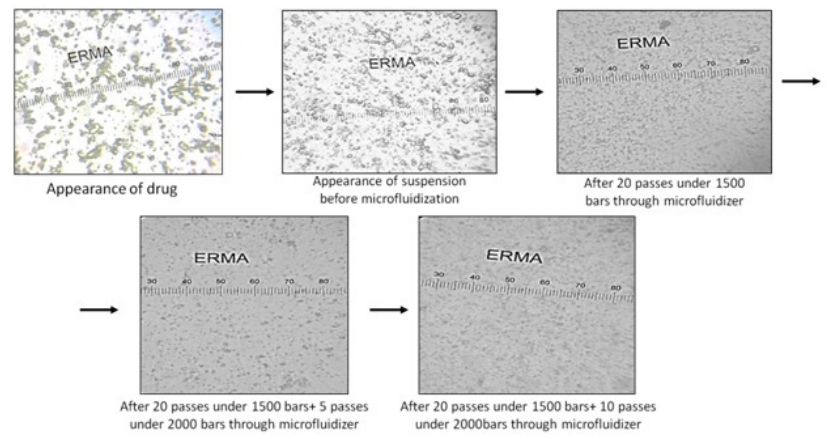

Figure 5: Microscopy images (45x) of size reduction of particles after processing through microfluidizer.

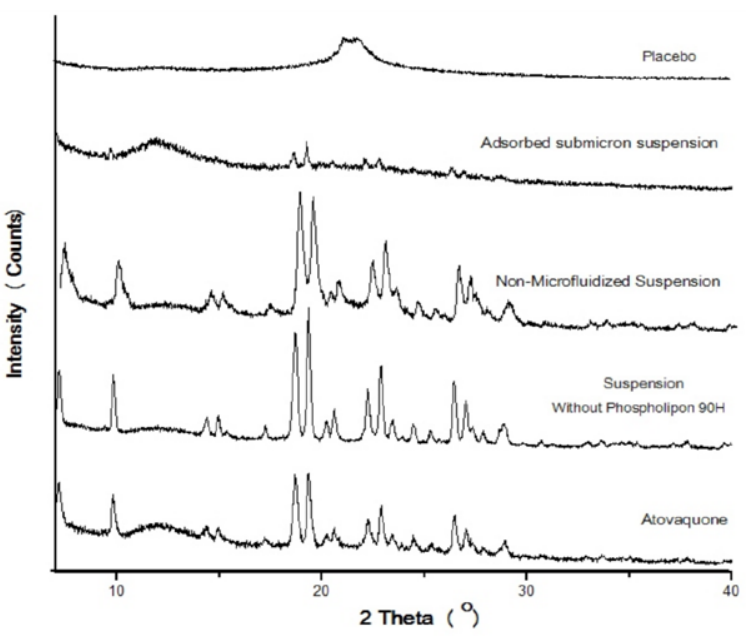

Figure 6: Comparative X-ray diffractogram of adsorbed submicron suspension. 


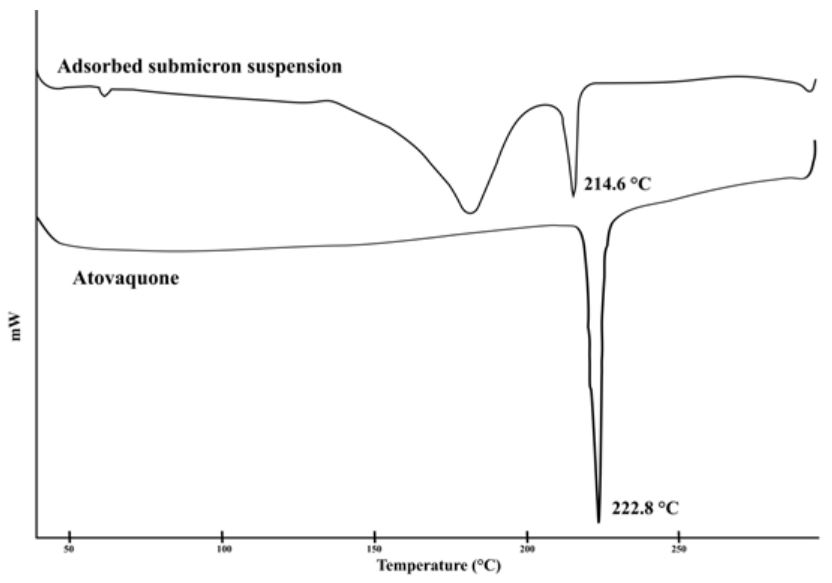

Figure 7: DSC thermogram of pure atovaquone and adsorbed submicron suspension.

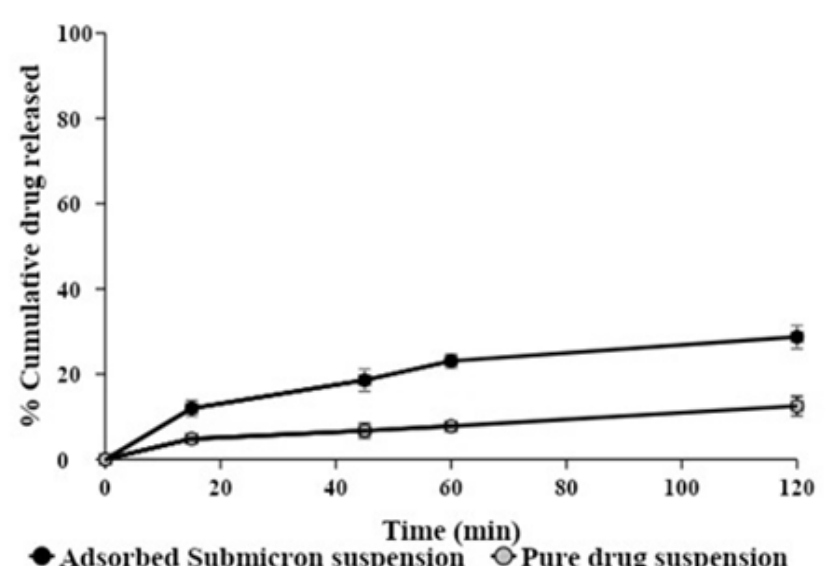

Figure 8: Comparative dissolution profile of adsorbed submicron suspension with a pure drug suspension.

of the original micronized suspension. Hence although polymeric surfactants can stabilize nanoparticles in an aqueous environment, their stabilization ability is compromised in the dehydrated state. It should be noted that there was an absence of larger particle aggregates after redispersion.

The XRD data of the drug was compared with the formulation containing only poloxamer 188 and poloxamer 188 with phospholipon $90 \mathrm{H}$ (Figure 6). The intensity of the peaks of non-microfluidized suspension (subjected to Ultra-Turrax and probe sonication) was lesser than the pure drug powder. The intensity of the peaks of adsorbed microfluidized micronized formulation was significantly reduced compared to that of the pure drug due to the dilution effect though there was no major change in the crystallinity of the drug.

The DSC thermogram of atovaquone after microfluidization and adsorption as shown in Figure 7 revealed an endothermic peak at $214.6^{\circ} \mathrm{C}$ while the pure drug shows a sharp endothermic peak at $222.8^{\circ} \mathrm{C}$. The slight reduction in melting endotherm temperature, $\mathrm{T}_{\mathrm{m}}$ compared with untreated drug suggests a decrease in crystallinity; however, the reduction was very small, which agreed with XRD analysis. The assay of the adsorbed powder was found to be $358.82 \mathrm{mg} / \mathrm{g}$.

The saturation solubility of atovaquone in water was found to be $0.2 \mu \mathrm{g} / \mathrm{ml}$ and saturation solubility of adsorbed micronized suspension was found to be 10.7 $\mu \mathrm{g} / \mathrm{ml}$. From the saturation solubility, it was seen that there was a drastic improvement in the solubility of the drug in micronized suspension as compared to the pure $\operatorname{drug}(p<0.05)$.

\section{In vitro dissolution studies}

The dissolution profile of the adsorbed microfluidized micronized suspension was compared to the unmilled pure drug suspension as shown in Figure 8. The adsorbed micronized suspension showed a release of around 30\% drug in $2 \mathrm{~h}$. The release showed a 2.3-fold times increase in the dissolution of the drug compared to suspension and drug $(p<0.05)$. Atovaquone in its crude unprocessed form is present as aggregates, which when processed through the three-stage top-down processing offers distinctly separated particles. Furthermore, the decrease in particle size increases the surface area of the particle and thus the dissolution. Poloxamer acts as a wetting agent which aids the drug to easily disperse in the media. It is postulated that further reduction in particle size of the micronized suspension will improve the dissolution.

\section{CONCLUSION}

Adsorbed micronized suspension of atovaquone was successfully optimized using $3^{2}$ full factorial design utilizing three concentrations of surfactant and three concentrations of stabilizer. The final optimized formulation containing $2 \%$ poloxamer 188 and $1 \%$ phospholipon $90 \mathrm{H}$ was microfluidized which successfully yielded micronized suspension. The stabilizers and the microfluidization process appeared to have the most significant impact on the quality of the micronized suspension. The adsorbed micronized suspension exhibited enhanced saturation solubility and in vitro dissolution as compared to untreated drug suspension. The micronized suspension was converted into solid by adsorption which can further be given as a dry syrup formulation with added sweeteners and flavors. The idea of size reduction of a rigid molecule like Atovaquone using microfluidization to improve its 
solubility provides a novel insight for the development of nanosuspension of atovaquone. ${ }^{20}$

\section{ACKNOWLEDGEMENT}

The authors are grateful to Glenmark Pharmaceuticals (Mumbai, India) for providing atovaquone and Lipoid $\mathrm{GmbH}$ (Germany) for providing Phospholipids $90 \mathrm{H}$. The authors further thank BASF Fine Chemicals (Mumbai, India) for providing gift samples of Kolliphor $^{\circledR}$ P188, Kollidon ${ }^{\circledR}$ CL SF and Kolliphor ${ }^{\circledR}$ EL. We thank Trident Equipments Pvt. Ltd., Mumbai for providing the facility for microfluidization of our samples. The authors also express their gratitude towards the management of Vivekanand Education Society's College of Pharmacy to provide the facility to conduct this research work.

\section{CONFLICT OF INTEREST}

The authors declare there is no conflict of interest.

\section{ABBREVIATIONS}

BCS: Biopharmaceutical Classification System; nm: nanometer; rpm: rotations per minute; PdI: Polydispersity index; HPLC: High Performance Liquid Chromatography; ANOVA: Analysis of Variance; USP: United States Pharmacopoeia; XRD: X-ray Diffraction; IPA: Isopropyl alcohol; OGD: Office of Generic Drugs; US FDA: United States Food and Drug Administration; DSC: Differential Scanning Calorimeter

\section{REFERENCES}

1. Rolan P, Mercer A, Weatherley B, Holdich T, Meire H, Peck R, et al. Examination of some factors responsible for a food-induced increase in absorption of atovaquone. Br J Clin Pharmacol. 1994;37(1):13-20.

2. Product Information Malarone ${ }^{\circledR}$ Tablets $(250 / 100)$ Malarone $®$ Junior Tablets (62.5/25) [Internet]. GlaxoSmithKline Australia Pty Ltd. 2016;16. [cited 2019 Jul 17]. Available from: https://au.gsk.com/media/306924/malarone-pi.pdf

3. Product Monograph: PrMALARONE $®$ PrMALARONE $®$ PEDIATRIC [Internet]. Glaxo Smith Kline Inc. 2015;47. Available from: https://ca.gsk.com/ media/591413/malarone.pdf
4. Serajuddin A. Solid dispersion of poorly water-soluble drugs: Early promises, subsequent problems and recent breakthroughs. J Pharm Sci. 1999;88(10):1058-66.

5. Borhade V, Pathak S, Sharma S, Patravale V. Formulation and characterization of atovaquone nanosuspension for improved oral delivery in the treatment of malaria. Nanomedicine. 2014;9(5):649-66.

6. Mohtar N, Khan NAK, Darwis Y. Solid Lipid Nanoparticles of Atovaquone Based on 24 Full-Factorial Design. IJPR. 2015;14(4):989-1000.

7. Calvo J, Lavandera J, Agüeros M, Irache J. Cyclodextrin/poly(anhydride) nanoparticles as drug carriers for the oral delivery of atovaquone. Biomed Microdevices. 2011;13(6):1015-25.

8. Cauchetier E, Fessi H, Boulard Y, Deniau M, Astier A, Paul M. Preparation and Physicochemical Characterization of Atovaquone-Containing Liposomes. Drug Dev Res. 1999;47(4):155-61.

9. Sordet F, Aumjaud Y, Fessi H, Derouin F. Assessment of the activity of atovaquone-loaded nanocapsules in the treatment of acute and chronic murine toxoplasmosis. Parasite. 1998;5(3):223-9.

10. Afifi SA, Hassan MA, Abdelhameed AS, Elkhodairy KA. Nanosuspension: An Emerging Trend for Bioavailability Enhancement of Etodolac. Int J Polym Sci. 2015;2015:16.

11. Seok SH, Kang S, Seo J, Kim S, Hwang K, Park E. Formulation of Nanoparticle Containing Everolimus Using Microfluidization and FreezeDrying. Chem Pharm Bull. 2016;64(10):1445-9.

12. Eerdenbrugh BV, Mooter GVD, Augustijns P. Top-down production of drug nanocrystals: Nanosuspension stabilization, miniaturization and transformation into solid products. Int J Pharm. 2008;364(1):64-75.

13. Gumaste SG, Pawlak SA, Dalrymple DM, Nider CJ, Trombetta LD, Serajuddin ATM. Development of Solid SEDDS, IV: Effect of Adsorbed Lipid and Surfactant on Tableting Properties and Surface Structures of Different Silicates. Pharm Res. 2013;30(12):3170-85.

14. Kathpalia $\mathrm{H}$, Salunkhe $\mathrm{S}$, Juvekar $\mathrm{S}$. Formulation of gastroretentive sustained release floating in situ gelling drug delivery system of solubility enhanced curcumin-soy lecithin complex. J Drug Deliv Sci Technol. 2019;53.

15. Kumar A, Singh D, Mathur P, Nellithanath TB, Sahal GP, et al. Pharmaceutical composition. India: United States; US 9,492.406 B2. 2016;1-28.

16. Fontana F, Figueiredo P, Zhang P, Hirvonen JT, Liu D, Santos HA. Production of pure drug nanocrystals and Nano Co-crystals by confinement methods. Adv Drug Deliv Rev. 2018;131:3-21.

17. Dearn AR. Atovaquone Pharmaceutical Compositions. United States; US 6649659 B1. 2003.

18. Gora S, Mustafa G, Sahni JK, Ali J, Baboota S. Nanosizing of valsartan by high pressure homogenization to produce dissolution enhanced nanosuspension: Pharmacokinetics and pharmacodyanamic study. Drug Deliv. 2016;23(3):93040.

19. Chaubal MVCP. Conversion of Nanosuspensions into Dry Powders by Spray Drying: A Case Study. Pharmaceutical Research. 2008;25(10):2302-8.

20. Kathpalia $\mathrm{H}$, Juvekar S, Shidhaye $\mathrm{S}$. Design and in vitro Evaluation of Atovaquone Nanosuspension Prepared by pH Based and Anti-solvent Based Precipitation Method. Colloid Interface Sci Commun. Elsevier. 2019;29:26-32. Available from: https://doi.org/10.1016/j.colcom.2019.01.002 
PICTORIAL ABSTRACT

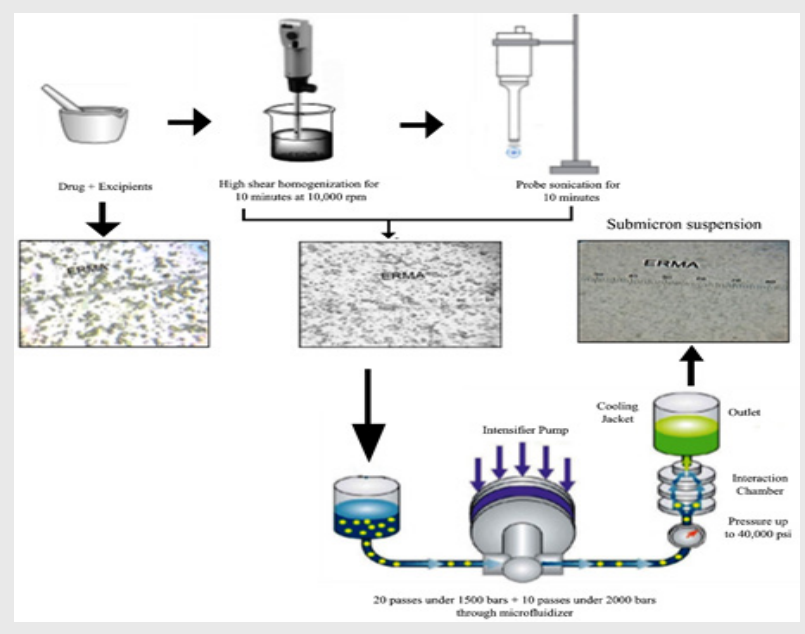

\section{About Authors}

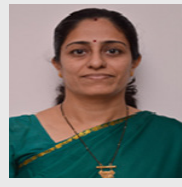

Harsha Kathpalia, Assistant Professor, VES College of Pharmacy, Mumbai. Specialization in formulation development. Total professional experience 22 years.

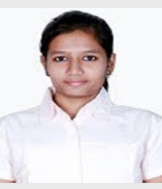

Sukanya Das, M.Pharm. student (2017), VES college of Pharmacy, Formulation scientist

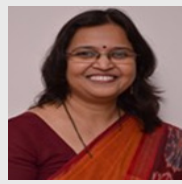

Dr. Supriya Shidhaye, Principal and Professor, VES College of Pharmacy, Mumbai. Total professional experience 28 years.

\section{SUMMARY}

Atovaquone is currently available as a tablet and oral suspension, both of which are required in large doses due to bioavailability constraints. The developed formulation highlights the efficiency of processes like sonication and microfluidization to reduce the particle size and improve the dissolution characteristics of Atovaquone.

Cite this article: Kathpalia H, Das S, Shidhaye S. Formulation and Optimization of Atovaquone Micronized Suspensions by Top-down Method. Indian J of Pharmaceutical Education and Research. 2021;55(1):77-85. 